\title{
Design and implementation of enterprise information management
}

\section{system and dynamic cost management}

\author{
LinHuaGAO, , a, Min LIN² \\ ${ }^{1}$ College of Computer Science and Technology,Wuhan University of Science and Technology, \\ Wuhan City, Hubei Province, China \\ ${ }^{2}$ Wuhan Engineering Institute, Wuhan City, Hubei Province, China \\ ahuazaizai0322@163.com
}

\begin{abstract}
Keywords:Business Management; MIS; Dynamic Cost Management; Cost Forecasting.
Abstract. With the rapid development of small and medium enterprises, cost management has become an important part of enterprise management. As the manufacturing enterprises are always faced with the volatility and uncertainty of the market, thus it puts forward new requirements for the timeliness of the product cost calculation. The realization of the dynamic cost management method is helpful for the enterprise to carry on the cost analysis, the cost forecast and the cost control in real time. In this paper, through the analysis of the needs of enterprise cost management, the original static cost management model has been transformed into a model of dynamic management function, which is better applied to the cost management of a production enterprise.
\end{abstract}

\section{Introduction}

With the development of economic globalization and integration, all kinds of manufacturing enterprises are facing increasingly fierce competition in international and domestic markets. strengthen the project cost management, increase the economic efficiency of enterprises, it is an inevitable choice to enhance the market competitiveness of enterprises and achieve long-term healthy development of enterprises.

Enterprises to improve profits mainly through two ways: to increase corporate income or control the cost of business expenses. Due to the influence of the business environment, market environment and other factors, the former is relatively difficult to control, so most companies choose to control the cost of the enterprise cost management.

Dynamic cost management is different from traditional cost management, it is a kind of real time replacement cost is based on the actual price of current market forecast material and current public cost, with the change of market environment changes.

In the advantage of enterprise dynamic cost management, can make the enterprise at any time in the production of the whole period can be time to understand and grasp the dynamic changes of the cost, so that enterprises can in the first time to appear the actual cost differences are analyzed. The dynamic cost management system should help the enterprise managers to proactively forecast the change trend of the product cost factors before and after the product is put into production, and can take effective measures and adjust the plan for the change of these factors.

This paper introduces the dynamic cost management method of small and medium-sized enterprises by taking a high-tech enterprise which produces new materials and refractory bulk materials. 


\section{requirement analysis}

According to the actual situation of refractory industry and enterprises and the actual needs of enterprises, the system is divided into six subsystems, as shown in Figure 1. Respectively, for the sales subsystem, procurement subsystem, production subsystem, quality inspection subsystem, human resources subsystem and dynamic cost subsystem. Most of the data of each subsystem is the source of the statistical data of the dynamic cost subsystem, which provides the basis for the final analysis and decision.

Based on the practical and advanced system of the principle of combining, through research and demonstration, the system should satisfy several basic requirements: (1)The system has the outstanding function of cost control, which is mainly embodied in the specific function modules such as cost forecasting, cost monitoring, cost warning and so on; (2) The system must have high safety and reliability; (3) The system should have good expansibility; (4) The system can collect the technical data and economic data of production and operation automatically and timely, and focus on processing; (5) For the basic cost data, the system should automatically monitor it in real time; (6) The system should establish a model library for quota revision and cost forecasting, which can automatically complete quota modifications and cost forecasts.

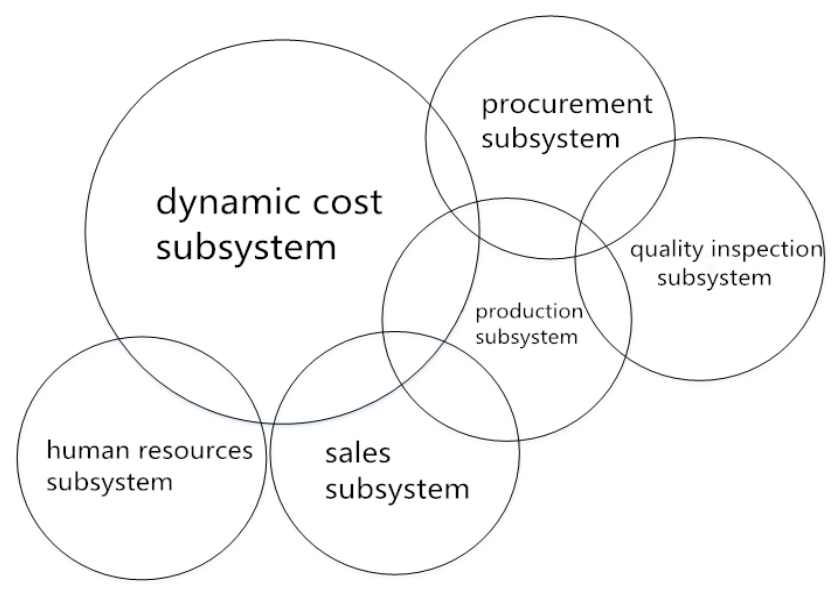

Fig. 1. System sub-function and its relation diagram

\section{System Technology Architecture}

Based on B / S mode, the system is developed using ASP.NET MVC4.0.0, and the asynchronous mechanism based on Ajax is used to process the page asynchronously. The system according to ASP.NET MVC4.0.0 divided into DAL, Views, BLL three layer structure, The overall system architecture shown in Figure 2.

DAL corresponding to the Model of the MVC, mainly used to deal with the relationship between entities in the system and its data related database operations, usually in the name of Repository end. View corresponding to the view of the MVC, it is responsible for all web interface display, relative to the system, the general DAL layer involves at least four related web interface, Create.aspx, Edit.aspx, Details.aspx , Index.aspx, that is, create the interface, editing interface, detailed interface and the index interface. BLL corresponding to the Controller of the MVC, responsible for business logic processing and data transfer between views, the general process is to read data from the DAL layer, through the BLL layer of data manipulation, it will be displayed in the Views corresponding to the web interface. 


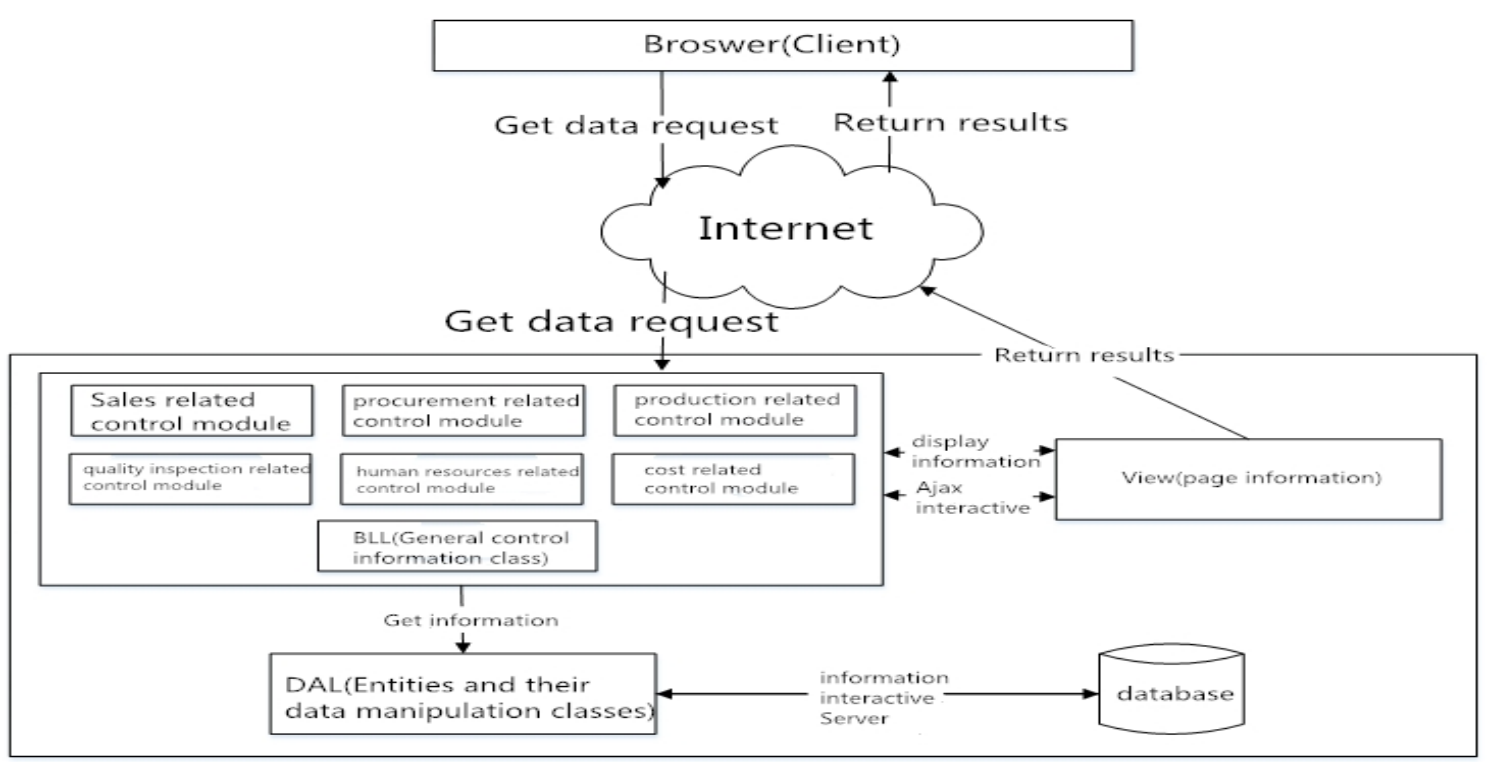

Fig. 2 . system technology architecture diagram

The system uses the MVC architecture model with the following advantages:

(1) low coupling.Use the hierarchical model to separate the role responsibilities from the business, when any module logic needs to be modified without the need for the overall process of restructuring, but only need to adjust some of the code.

(2) conducive to engineering management. Different layers perform their duties, each layer code similarity is strong, is conducive to engineering and Instrumentalism manage program code, When a replacement is required, a solution can be generated automatically after a simple replacement.

(3) Easy deployment. MVC model development will save a considerable part of the duplication of work, by the system according to the template to generate the corresponding controller class, database operation class, the developer can focus on the business logic processing.

\section{System detailed design}

The system is based on various departments, but also can be subdivided into subsystems, each sub-system including the related sub-function flow, the overall function of the system shown in Figure 3. the system including DAL layer, includes more than and 260 kinds of entity class, business logic class and data operation class, and the database table is more than and 150. Here I only focus on the dynamic cost subsystem, main functions of dynamic cost subsysteminclude: cost accounting, costdynamic comparison and cost dynamic prediction of the three modules. 


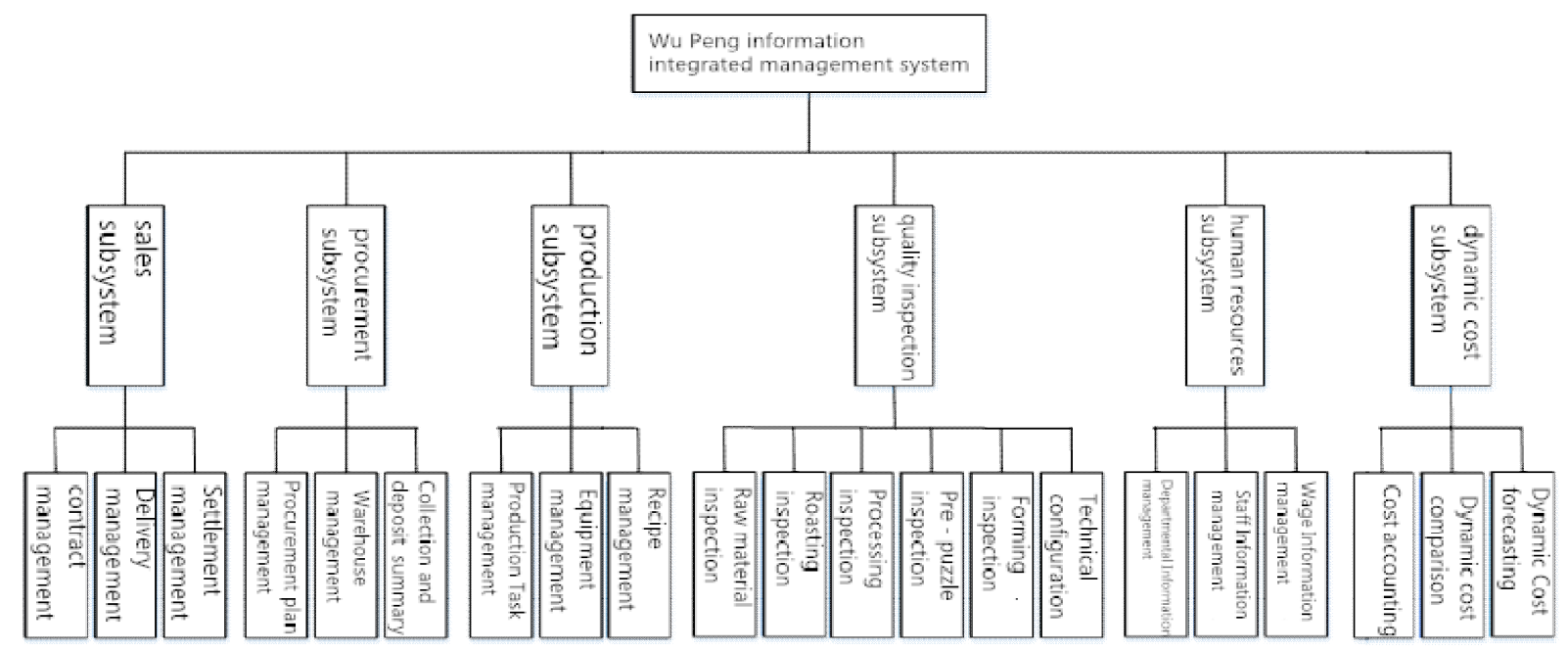

Fig.3. system overall function diagram

\section{System implementation}

This article mainly introduces the function and implement method of dynamic cost management system.

\section{A. Cost accounting}

First, select the cost control module from the list on the left side of the main interface of the system. The drop-down list includes production management, sales management, and cost management. Figure 4 for the raw material cost accounting interface.

Raw material cost accounting includes basic creation, deletion, detailed, query and report export function. The cost accounting statements of other workshops are roughly the same as the raw material cost accounting statements, so is omitted here.

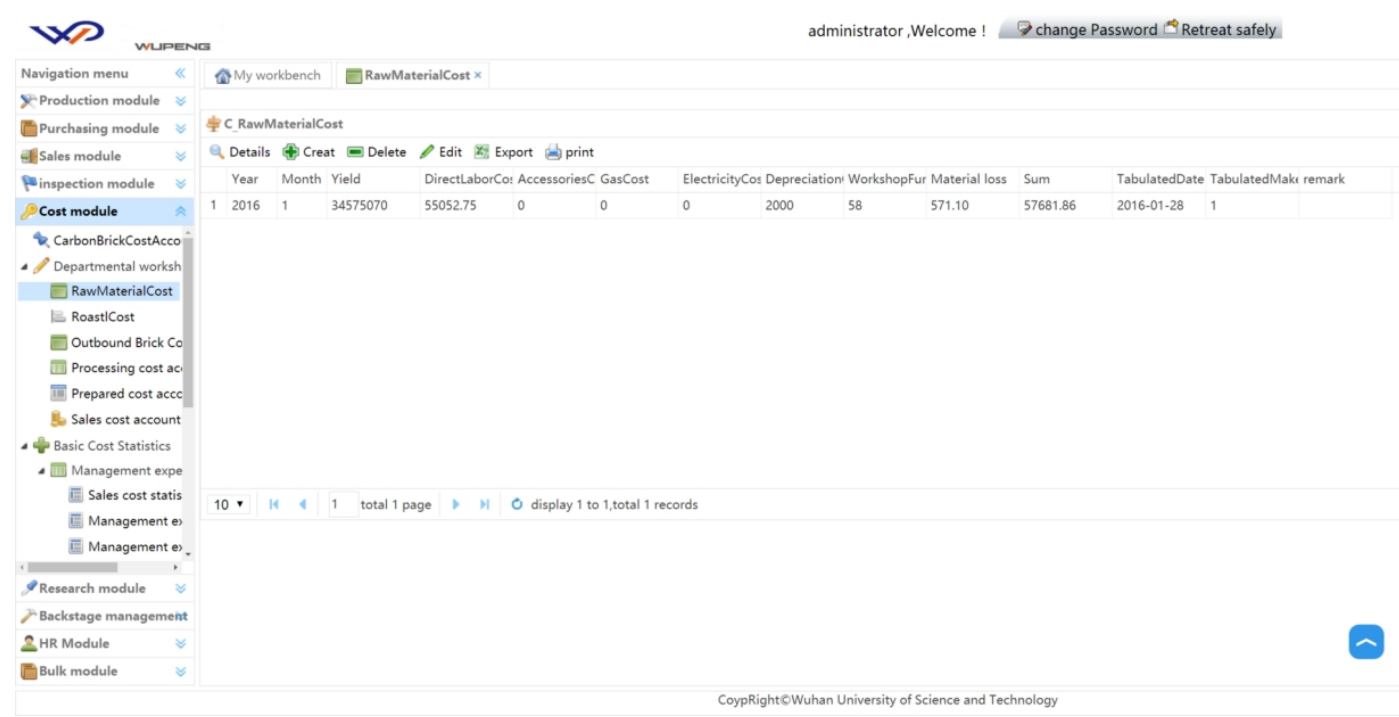

Fig.4. for the raw material cost accounting.

\section{B. cost dynamic comparison}

The cost comparison subsystem is in the cost management subsystem. The comparison process includes year-on-year data, month-on-month data, and overall cost comparison. Click the cost 
comparison in the cost control subsystem, click the month-on-month data, the system will display the company's current month consumption cost data and last month consumption cost data comparison chart. Each sub-system in the figure will be differentiated by different colors, and in the upper left shows the type to which they belong. The mouse will be placed on the corresponding pie chart will show the corresponding percentage of the total cost of the subsystem, as shown in Figure 5.

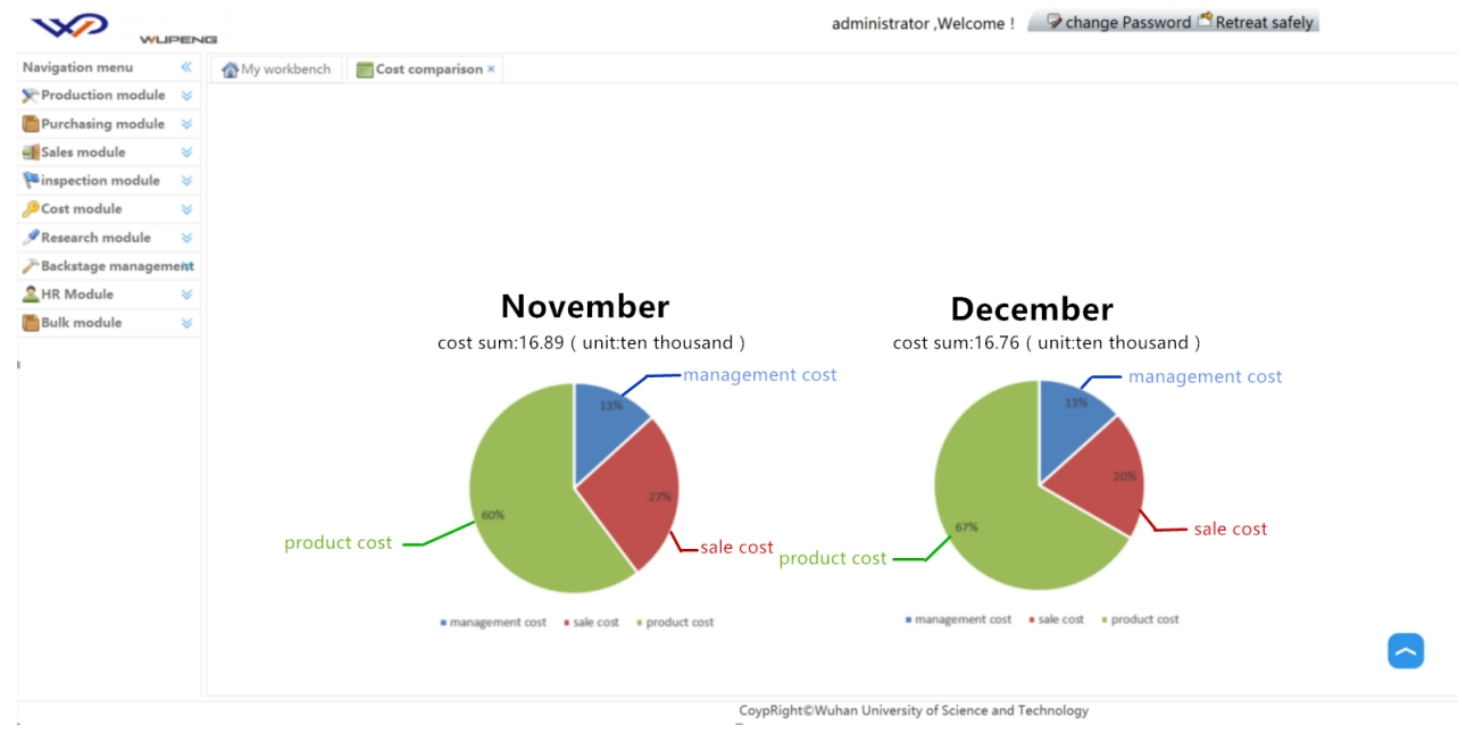

Fig.5. month-on-month cost data

When you click on the year-on-year data, enter the cost of the first level interface. The system will display the company this month the total cost of consumption and consumption data in the same month last year the total cost data comparison chart.

If you want to view the details of a single subsystem in the cost subsystem, click the pie segment of the pie chart to enter the cost comparison second level interface, which will display the detailed data of the sector.

The overall cost comparison can also be compared to items of the overall data, select the start time and the end time of the cost data, the system will be displayed according to effect of Cost classification .

\section{Dynamic cost forecast}

In the sales management module, click the contract estimate to enter the main interface of the contract estimate, the main interface includes the creation of the estimated contract, create the estimated product details, generate forecast results, view the contract estimate. First click on the creation of the estimated contract button to create the estimated contract, the system pops up a window, Then fill in the contract unit and the area.

After creating the estimated contract, click the Create Estimate Detail button to add the estimated product details to the estimated contract, including the product name, product quantity, model number, estimated contract number, etc. Click Create, the contract cost estimate was created successfully.

After the estimated basic information is added, click the Generate Estimate Effect button. The estimated price and estimated total price of the products in the corresponding contract will be estimated based on the information stored in the system, and display the results.

Cost forecasting can also forecast the total cost, including production cost forecasting, sales cost forecasting, management cost forecasting, production cost forecasting as an example, the choice of 
forecasting start time and end time, the system will combine the relevant forecasting methods to dynamically generate production costs prediction scheme, as shown in figure 6 .

\section{Dynamic cost warning}

The system also carries out dynamic monitoring on material cost, production cost, cost of sales, management cost and so on, the relevant personnel can set the warning threshold according to the requirements of each relevant cost, when a data exceeds a threshold value, the system will automatically mark the corresponding product information, at the same time to remind the relevant personnel costs already exceed the expected cost of the current.

For example, when the system implemented dynamic monitoring on the price of finished goods of sales cost, the warehouse manager can be set according to the requirements of a variety of finished products price warning threshold, when a current market price of finished products is less than the threshold, the system automatically

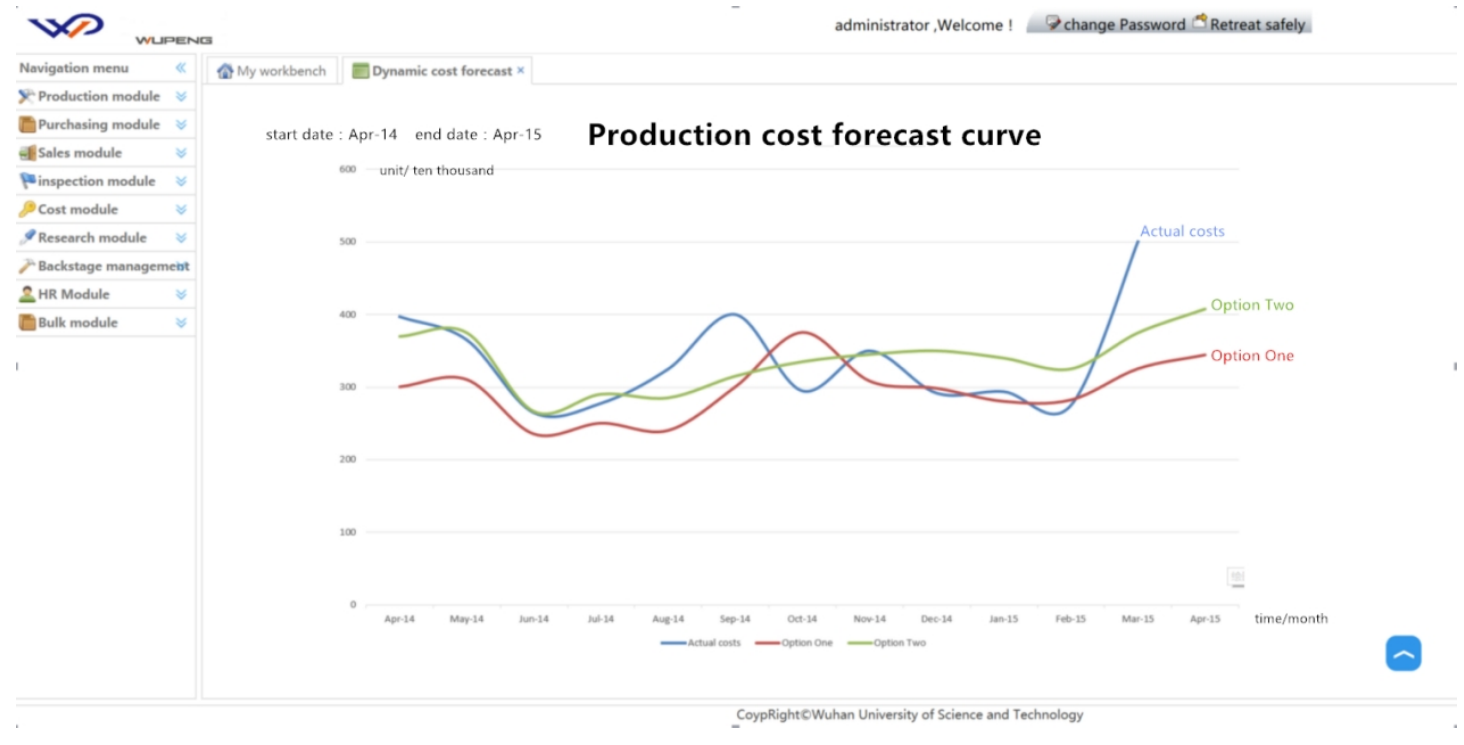

Fig.6. production cost forecast curve

remind the warehouse manager that the current sales price of finished products does not reach the expected selling price.

The system also implements the dynamic monitoring of the expenses of the various departments When a department's expenditure exceeds the expected cost, the system will warn the department. When the administrator accesses the system, it will receive an alert pop-up prompt.

\section{Concluding remarks}

The dynamic cost management system of manufacturing enterprise can effectively manage and control the cost of the enterprise, and the contradiction between input, output and benefit should be a basic point of view. Scientific and effective dynamic cost management can play a significant role in the control of manufacturing costs, reduce costs and increase profits. This article aims at the actual needs of a business management, makes a preliminary exploration of the dynamic cost management system, and transforms the original static cost management model into a model with initial dynamic management function, which plays a good role in the cost management of the enterprise.

\section{References}

[1] Wu Xichun. The New Stage of China 's Steel Industry Development [J]. China 's steel industry, 2015(1):6-15. 
[2] Cheng Yunxue. Study on the effect of energy saving and emission reduction in thermal power enterprises[D]. North China Electric Power University,2013.

[3] Li Hongxia. Development of China's refractory industry and its resources, energy and environment[J]. Refractory material, 2010(6):401-403.

[4] Zhang Rumin. Risk Control in ERP Implementation[D]. Southeast University, 2009.

[5] Gong Ming. Analysis on the current situation and application of ERP software in China[J]. China new communications, 2015(9):15-16.

[6] Niu Hao. Research on customer relationship management of steel refractory company[D]. Southwest University of Science and Technology, 2014.

[7] Li Li. Research on effective countermeasures of enterprise cost management[J]. China Economic and Trade ,2014(8):210-211.

[8] Zhang Feng. Dynamic Cost Management of Real Estate Enterprise Project[J]. Research on Urban Construction Theory, 2015(21).

[9] Xiande Z,Fujun L,Scott Young. A study of Manufacturing Resources Planning (MRPII) implementation in China[J]. International Journal of Production Research, 2002, Vol.40 (14): 3461-3478.

[10] Huang Wenxiao. Design and Realization of Real Estate Target Cost Management System Based on .NET Technology[D]. Tianjin University,2013.

[11] Xiaole L,Lianggang N, Jianrong Y. Formal Construction of Secure Information Transmission in Office Automation System[J]. Web information systems and mining. WISM 2012.

[12] Li Min. Problems and Countermeasures of accounting cost accounting[J]. Research on Urban Construction Theory, 2013(10).

[13] Choi Youngbin,Saqqur Maher,Stewart Eileen. Relative energy index of microembolic signal can predict malignant microemboli.[J]. Stroke,2010,414.

[14] Sheng Jianrong. Realization of computer cost accounting system for small and medium sizedmanufacturing enterprises[J]. Automation technology and Application, 2014(7):27-33. 